\title{
Lagrangian Transport of Particles in Ria de Aveiro Lagoon, Portugal
}

\author{
J. M. Dias' 1 , J. F. Lopes ${ }^{1}$ and I. Dekeyser ${ }^{2}$ \\ 'C.Z.C.M. - Dept. Física, Universidade de Aveiro, 3810-193 Aveiro, Portugal \\ ${ }^{2}$ C.O.M., Université de la Méditerranée, 13288 Marseille Cedex 09, France
}

Received 24 April 2000; accepted 28 August 2000

\begin{abstract}
A two-dimensional vertically integrated hydrodynamic model coupled to a particle tracking model is applied to study the dispersion processes and residence time in Ria de Aveiro lagoon (Portugal).

The only dispersion process that is considered in this study is the advection, according to the main characteristics of the local hydrodynamic. The particle tracking model computes the particles position at each time step, using a fourth-order Runge-Kutta integration method. The dispersion of passive particles released along the lagoon and in critical areas are studied in this work. The residence time is also determined for the entire lagoon.

The results show that the mixture between particles coming from different channels of the lagoon is negligible in a time scale higher than 2 tidal cycles. The residence time for the lagoon central area is about 2 days, revealing a strong marine influence in this area. At the upper reaches of the channels were found values higher than 2 weeks. 12001 Elsevier Science Ltd. All rights reserved
\end{abstract}

\section{Introduction}

A lagrangian model constitutes a powerful tool for studying dispersion processes in coastal waters, estuaries and lagoons. It allows analysis of dispersion of water masses, represented as passive particles, and estimation of residence times at a low computational cost compared to eulerian models. Those models provide considerable time saving, because they only follow the track of the selected particles, in contrast with eulerian transport models, which calculate the concentration of any property anywhere in the simulation domain. The main advantage of a fully lagrangian formulation over eulerian transport models is its higher accuracy in describing localized emission spots avoiding artificial numerical diffusion in presence of strong gradients. By these reasons

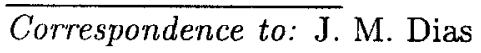

these models have been widely used recently to study the transport of passive particles in coastal areas (Hofmann et al., 1991; Joseph et Swathi, 1999; Gomez-Gesteira et al., 1999; Brooks et al., 1999).

The use of vertically integrated two-dimensional models was generalized over the last decades to study shallow areas with strong vertical mixture (Falconer and Owens, 1990; Cheng et al., 1993; Marinone, 1998). In spite of the improvement of three-dimensional models during the last decade, two-dimensional models still remain useful to describe situations with a weak vertical stratification. They are specially used to study wave phenomena such as tides and tidal currents, or transport of solutes for which effective dispersion coefficients can be determined empirically. Ria de Aveiro physical and geometric characteristics makes these kind of models ideal to study its hydrodynamic behaviour.

The purpose of this work is to study the dispersion and mixture processes and to determine the residence time for Ria de Aveiro lagoon using the transport of passive particles induced by the tidal flow. Some particles are released in critical areas, as harbours and shipyards, and their tracks due to the tidal currents are also determined. This work is performed coupling a particle tracking model to a previous calibrated twodimensional hydrodynamic model and is carried out under no wind and river discharge conditions, corresponding to the longest residence time.

\section{Study Area}

Ria de Aveiro is a very important area in the Portuguese coast. It provides natural conditions for harbour, navigation and recreation facilities and it is also a place of discharge of domestic and industrial wastes. It offers good conditions for agricultural development along its borders and for the set up of a large number of small and medium industries. 


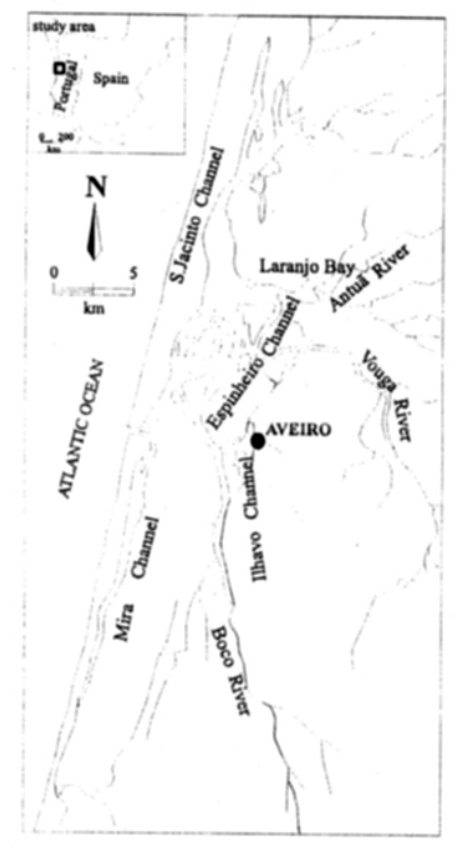

Fig. 1. Geographic map of Ria de Aveiro.

Ria de Aveiro is a shallow coastal lagoon (Fig.1), located on the Northwest Atlantic coast of Portugal $\left(40^{\circ} 38^{\prime} \mathrm{N}, 8^{\circ} 44^{\prime} \mathrm{W}\right)$. It is formed by a complex dendritic pattern of channels characterised by the existence of significant intertidal zones, namely mud flats and salt marshes. It is connected with the Atlantic Ocean through an artificial channel at the west side and it exchanges most part of its water with the ocean by tidal input across this narrow entrance.

The lagoon has a maximum width and length of 10 and $45 \mathrm{~km}$, respectively, and in a spring tide covers an area of $83 \mathrm{~km}^{2}$ at high tide, which is reduced to 66 $\mathrm{km}^{2}$ at low tide. The average depth of the lagoon is about $1 \mathrm{~m}$, but in the channel located near the mouth the depth is around $20 \mathrm{~m}$. The other navigation channels, where dredging operations are frequently carried out, are about 7 m deep. This strongly variable depth, associated with the large number of narrow channels and with the existence of intertidal areas makes the numerical modeling of Ria de Aveiro a difficult task.

The lagoon has four main branches radiating from the sea entrance (Fig.1): Mira, S.Jacinto, Ilhavo and Espinheiro channels. The Mira channel is an elongated shallow arm, with $20 \mathrm{~km}$ length. S.Jacinto channel is about $29 \mathrm{~km}$ long and is the most important channel concerning average width and length. Ihavo channel is the narrower and shorter of the main channels, with a length of $15 \mathrm{~km}$. Finally, Espinheiro is a short channel with about $17 \mathrm{~km}$ length which give a complex nctwork of small dead arms.

The tide at the mouth of the lagoon is predominantly semidiurnal (tidal form number, $\mathrm{F}=0.102$ ), with a mean tidal $r$ ange of about $2.0 \mathrm{~m}$. It propagate from the mouth as a mixed progressive and standing wave and is present in the entire lagoon. Tide generates strong currents in deep and narrow channels, but not in the intertidal area. The highest values are found at the beginning of Espinheiro and S.Jacinto channels, and specially in the entrance channel, where the tidal current amplitude can be higher than $1 \mathrm{~m} / \mathrm{s}$ (Dias et al., 2000). The induced tidal residual currents are directed downstream in almost all the domain because the ebb tidal currents are higher than the flood ones in this lagoon (Dias et al., 1998).

\section{Model}

\subsection{Hydrodynamic model}

In order to study the transport processes in a situation of low river flow and negligible wind stress was used a two-dimensional vertically integrated model (Leendertse and Gritton, 1971). This barotropic hydrodynamic model solves the well known shallow water equations:

The simulation of the flow in a complex domain like Ria de Aveiro implies the use of the finest possible grid. For the hydrodynamic model, a rectangular computational grid with 160 cells in the $x$-direction (eastward) and 393 cells in the $y$-direction (northward), was the finest possible (the cells measure $100 \times 100 \mathrm{~m}$ ). With this grid the narrower channels are represented with its width exaggerated, but conserving its water volume.

The horizontal eddy viscosity is considered to be constant in time and space $\left(20 \mathrm{~m}^{2} / \mathrm{s}\right)$. The bottom stress was calculated using a quadratic law (Dronkers, 1969) and the Manning-Chezy formulation. The Manning rugosity was adjusted through the model calibration, and were determined different values for each channel.

At the open boundary was imposed the elevation over the reference level, determined using the tidal harmonics constituents provided by the Hydrographic Institute of the Portuguese Navy. The initial conditions were a horizontal level and null velocity in all the grid points. Along the solid boundaries was imposed a null normal velocity and was assumed a free slip condition, since the grid is not fine enough to simulate the horizontal boundary layers.

\subsection{Particle tracking model}

The two-dimensional trajectories of the simulated particles are computed using a Lagrangian approach, solving the equation:

$$
\begin{aligned}
X_{\imath}\left(x_{0}, y_{0}\right)^{n+1}= & X_{\imath}\left(x_{0}, y_{0}\right)^{n} \\
& +\int_{t_{0}+n \Delta t}^{t_{0}+(n+1) \Delta t} u_{\imath}(x, y, t) d t
\end{aligned}
$$


at each time step. $X_{\imath}\left(x_{0}, y_{0}\right)^{n+1}$ is the position at the instant $n+1$ of the particle released at the point $X_{\imath}\left(x_{0}, y_{0}\right)^{0}$ The time integral is computed using a fourth-order Runge-Kutta scheme:

$K_{1 \imath}=\Delta t \times u_{\imath}\left[t, X_{\imath}\left(x_{0}, y_{0}\right)^{n}\right]$

$K_{2 \imath}=\Delta t \times u_{i}\left[t+\frac{\Delta t}{2}, X_{2}\left(x_{0}, y_{0}\right)^{n}+\frac{K_{1 i}}{2}\right]$

$K_{32}=\Delta t \times u_{2}\left[t+\frac{\Delta t}{2}, X_{\iota}\left(x_{0}, y_{0}\right)^{n}+\frac{K_{2 i}}{2}\right]$

$K_{4 \imath}=\Delta t \times u_{\imath}\left[t+\Delta t, X_{2}\left(x_{0}, y_{0}\right)^{n}+K_{3 i}\right]$

$$
\begin{aligned}
X_{\imath}\left(x_{0}, y_{0}\right)^{n+1} & =X_{\imath}\left(x_{0}, y_{0}\right)^{n} \\
& +\frac{K_{1 i}}{6}+\frac{K_{2 i}}{3}+\frac{K_{3 i}}{3}+\frac{K_{4 i}}{6}
\end{aligned}
$$

where $X_{\imath}\left(x_{0}, y_{0}\right)^{n+1}$ represents the new location of a particle that is advected from its previous position $X_{\imath}\left(x_{0}, y_{0}\right)^{n}$ by the velocity $u_{i}=\left(u_{\imath}, v_{i}\right)$ in a time interval $\Delta t$, and $K_{j i}$ represents the Runge-Kutta coefficients.

The implementation of the above model is straightforward, provided that a velocity field, as the one simulated by the two-dimensional hydrodynamic model, is available. In reality particles may move in response to additional processes, such as sinking, diffusion and dissolution or aggregation. However, the dominant transport process in Ria de Aveiro is the advection.

The hydrodynamic model calculations are performed on a discrete grid in the horizontal plan but the simulated particles are free to move to any position between the grid points. Therefore, the model velocities must be interpolated to the instantaneous particle positions prior to the application of the time step equations $(2-6)$.

Since the model variables are arranged in a spacestaggered grid the $u_{\imath}$ and $v_{i}$ interpolations must be done separately. The interpolation is carried out considering the particle velocity at each point proportional to the a)

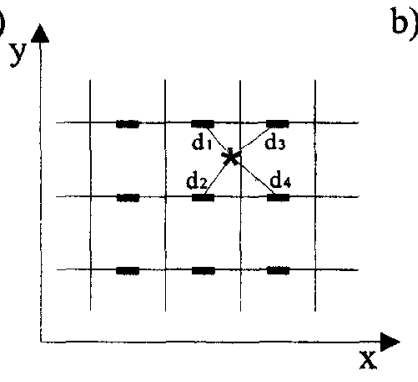

b)

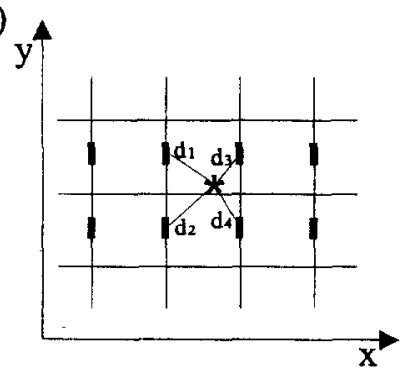

Fig. 2. Distance from the particle position to each one of the nearest velocity cells. a) $u_{\imath}$ interporlation; b) $v_{2}$ interpolation. computed velocities of the nearest four grid points (previously determined) weighted by the reverse of the distance from the particle position to each one of the grid points (Fig.2):

$u_{2}=\sum_{\jmath=1}^{4}\left(P_{\jmath \imath} U_{\jmath 2}\right) / \sum_{j=1}^{4} P_{\jmath 2}$

$v_{\imath}=\sum_{j=1}^{4}\left(P_{\jmath 2} V_{\jmath \imath}\right) / \sum_{j=1}^{4} P_{j \imath}$

where $P_{j i}=1 / d_{j i}$ and $d_{j i}=\sqrt{\left(x_{\imath}-x_{j}\right)^{2}+\left(y_{i}-y_{j}\right)^{2}}$.

Once the two velocity components are known at the particle locations, the particle locations are advanced forward in time by the fourth-order Runge-Kutta scheme.

Special attention should be paid to describing particle movement near the boundaries. The particles that move out of the computational domain through the open boundary are assumed to loose their identity and do not return. Finally, those particles crossing the solid boundary are relocated at their previous position.

\subsubsection{Model validation}

The accuracy of the particle tracking model was tested advecting a particle in a well-known velocity field, with characteristics similar to the ones found in Ria de Aveiro. The computed particle positions time evolution was compared with the equations analytical solution. The particles were advected by a two-dimensional periodic velocity field, with a strong semi-diurnal constituent and a weak diurnal one:

$$
\begin{aligned}
& u=A_{1} \sin \left(\omega_{1} t+\phi_{1}\right)+A_{2} \sin \left(\omega_{2} t+\phi_{2}\right) \\
& v=A_{3} \sin \left(\omega_{1} t+\phi_{3}\right)+A_{4} \sin \left(\omega_{2} t+\phi_{4}\right)
\end{aligned}
$$

with $A_{1}=4, A_{2}=0.32, A_{3}=3, A_{4}=0.12, \phi_{1}=$ $0, \phi_{2}=\pi / 18, \phi_{3}=\pi / 8, \phi_{4}=\pi / 10, \omega_{1}=2 \pi / T_{1}=$ $2 \pi /(12.25 \times 3600), \omega_{2}=2 \pi / T_{2}-2 \pi /(24.5 \times 3600)$.

The model computed particle trajectory comparison with the analytical solution trajectory is depicted in Fig.3. The trajectories are almost coincident which demonstrate the good accuracy of the model to compute the advection of passive particles in a periodic flow similar to the one characteristic of Ria de Aveiro.

\section{Results and Discussion}

The lagrangian particle tracking model was used to stu$\mathrm{dy}$ the dispersion of particles released in different lagoon channels, the advection of particles released in critical areas and to determine the lagoon residence time. 


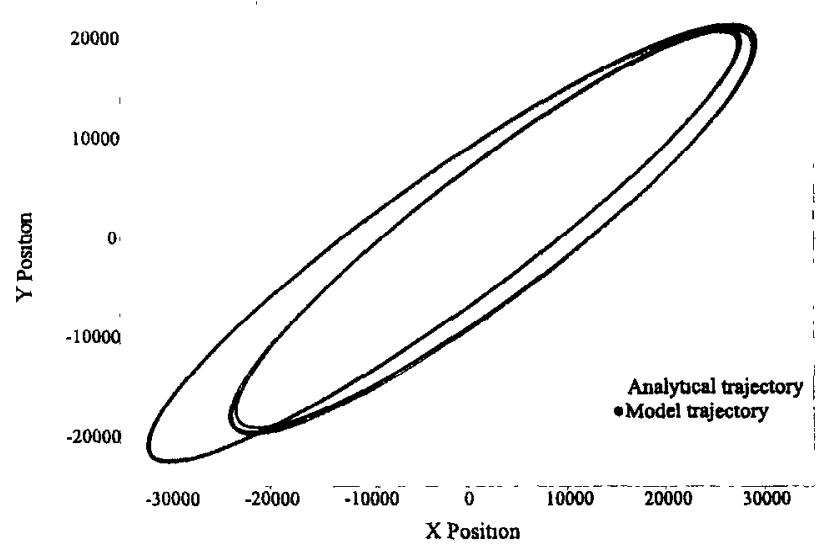

Fig. 3. Comparison between the trajectories determined by the Lagrangian model and obtained solving the analytical equations.

\subsection{Tidal mixing}

In order to study the mixture between particles released in the more important lagoon channels particles were emitted from each grid cell. The lagoon was divided in 6 different naturally delimited areas, corresponding each one to the main lagoon channels. Different colours were attributed to the particles released in each one of these areas. The particles were released at high tide at the lagoon mouth (beginning of ebb), so it is expected a net export of the particles situated near the mouth from the lagoon to the ocean.

Figure 4 shows the initial particles position and their position at the time of consecutive low and high water at the lagoon mouth. The displacement of the emitted particles is in accordance with the tidal residual circulation in the lagoon. The particles released at the central area are rapidly flushed out of the lagoon, whereas the ones released at the far end of the channels remain there throughout the calculation. This behaviour suggests that in these areas there are strong conditions to occur sedimentation. Other areas where particles tend to accumulate due to low tidal currents (e.g. Torreira southern zone, represented by light blue particles) are also identified as possible sedimentation areas.

The particles released at the middle area of Mira channel are trapped in this channel for a longer time than expected. During the ebb they are transported northward by the currents, but since these currents are weak in this area the ebb time is not enough to bring them to the entrance channel. In the next flood most of these particles are transported southward, coming again to the inner part of the channel. In this way the dark blue particles are moving back and forth in Mira channel and in each tidal cycle only a small part of these particles goes out of the lagoon.

The green particles rapidly go out of the lagoon because during the ebb they are transported to areas of high tidal velocity in S.Jacinto channel and in the begin- ning of Espinheiro channel. The northern green particles are advected through both the referred channels providing the only case of mixture of particles coming from different areas inside the lagoon.

The time evolution of the position of the the different colour particles reveal that almost there isn't mixture between particles from the different channels. Therefore each one of the channels can be considered independent. The particles coming from the different channels are mixed only in the entrance channel during the ebb, where they are rapidly flushed out due to the strong currents that exist here. In the next flood only a very small part of this water come again into the lagoon, because the water goes out as a narrow and strong jet and comes in from an area similar to a fan centered in the entrance channel. These characteristics of the water exchange between the lagoon and the ocean suggests a very good water renewal (after one tidal cycle almost there aren't yellow particles inside the lagoon).

\subsection{Particles tracks}

The tracks of particles released just as the flood begins at the lagoon mouth in four different areas of the lagoon are determined and depicted in Figure 5 ( lucations where the particles are released). These particles are released in areas where the pollution risk is high: 1 - highly polluted area due to the existence of medium industries; 2 - commercial harbour; 3 - new coastal fishing harbour; 4 - offward fishing harbour and shipyards. For each location the tracks of particles released in continuous grid cells are studied in order to illustrate the chaotic nature of the tidal flows in Ria de Aveiro (Fig. 5 a) and b)).

Although the particles in each situation were initially separated by only $100 \mathrm{~m}$ (the grid spacing), they soon diverged and followed different paths. The particles released at location 1 have completely different trajectories (green lines). In situation a) the particle is trapped in Laranjo bay, but in situation b) the particle is transported through several channels in a complex trajectory and is swept out of the lagoon. During the first tidal cycle the particle remains near Laranjo bay, but in the second one it is transported southward through Espinheiro channel during the ebb and northward during the flood. The ensuing ebb capture the particle and bring it southward through S.Jacinto channel and the next flood carries the particle back again through Espinheiro channel. The following ebb finally transports the particle to the ocean.

The trajectories of the particles released at the other locations are not so different from situation a) to situation b), however there are interesting features that must be pointed out. The particle released at location 2 has a longer path in situation a) and is entrained to the northern area of the lagoon through Espinheiro channel before being swept out of Ria de Aveiro. The particle 

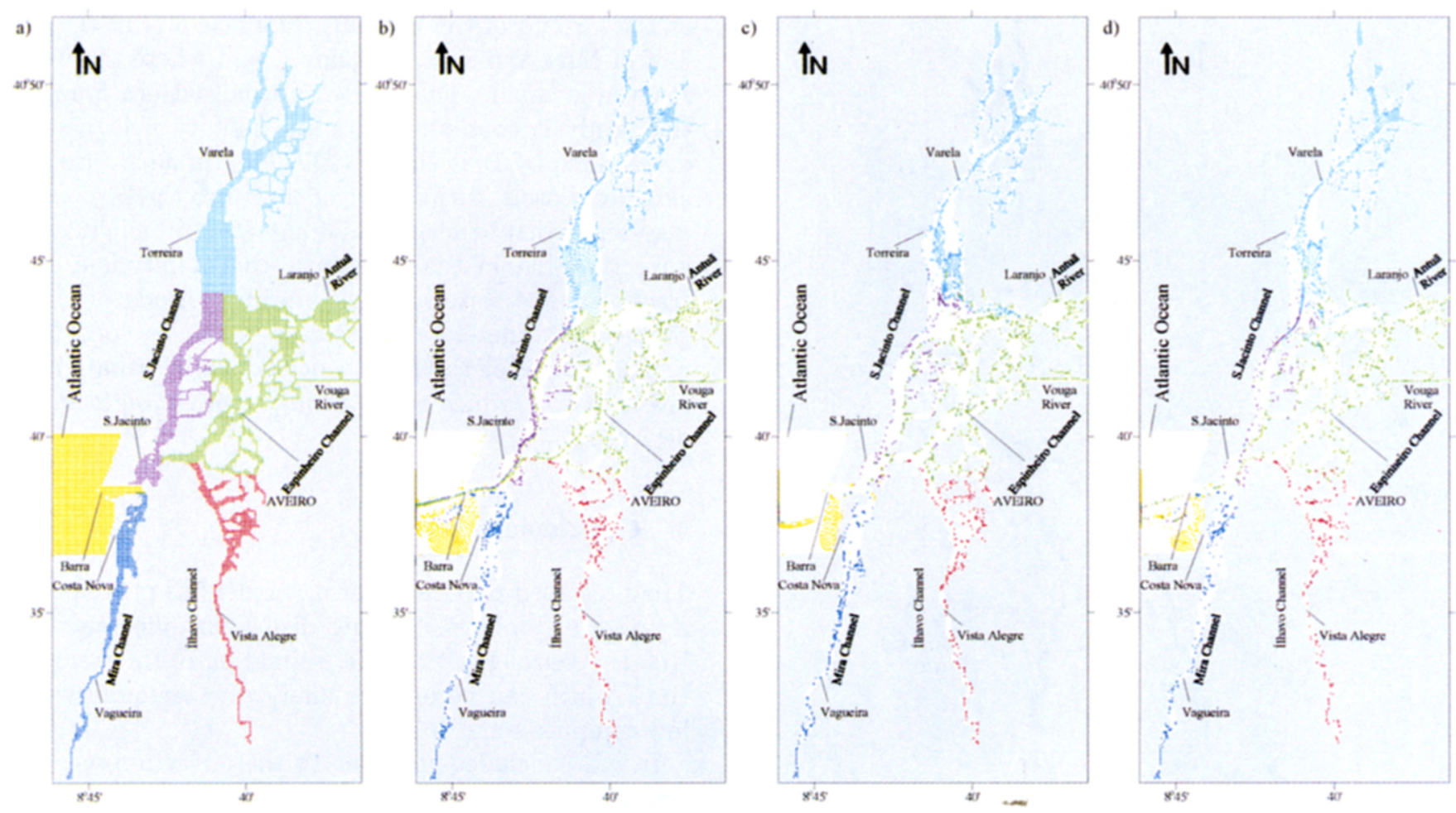

Fig. 4. Particles position time evolution sequence for particles released in each grid cell. The different times represented are referred to the lagoon mouth: a) high tide; b) low tide; c) high tide; d) low tide.

released at location 3 in situation a)is rapidly transported to the ocean, while in situation b) it is carried to the beginning of Espinheiro channel after being transported to the ocean and only in the ensuing ebb it definitively goes out of the lagoon. The particle number 4 has similar trajectories in both situations, but in case a) it is entrained closer to Aveiro in a longer path.

The sensitivity of the particles trajectory to their initial locations indicated the complex, nonlinear nature of the tidal regime. Such flow character is described as "Lagrangian chaos" by Zimmerman (1986), who shows that the chaotic motion can account for effective turbulent diffusion coefficients one or two orders of magnitude larger than otherwise expected.

Although the results obtained in section 4.1 reveal that almost there isn't mixture of particles from different channels after two tidal cycles, it is evident from the trajectories determined in this section that in shorter time scales there is important mixture between particles released in contiguous channels. Therefore, local episodes of pollution in each one of the critical areas considered in this study can also have important consequences in the adjacent channels, obviously depending on the tidal phase at the time of the event.

\subsection{Residence time}

To develop a useful map of residence time for the lagoon, model runs were seeded with particles released in
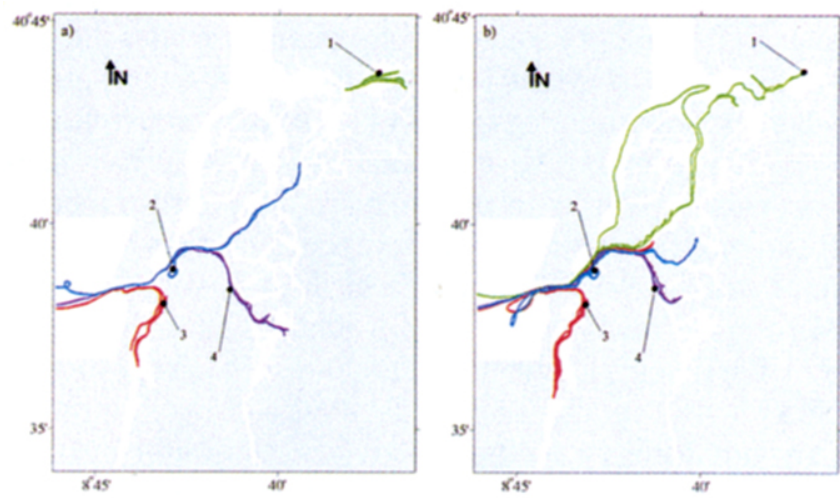

Fig. 5. Tracks of particles released in adjacent grid points (a) and b)) at four different areas of the lagoon ( $\bullet$ locations where the particles were released).

every grid cell at many different stages of the tidal cycle. The tidal-mean residence time determined in this way is shown in Figure 6. The contoured values were obtained by averaging over 12 separate model runs with releases at each lunar hour of the tidal cycle. For each run one particle was released at each grid cell and tracked for up to 14 days after a spin up period of about 3 days. Particles that were not flushed from the lagoon during each run were assigned a residence time of 14 days. It is important to recall that the influence of the wind and of the rivers freshwater input has not been included.

In almost all the lagoon, most particles escape re- 


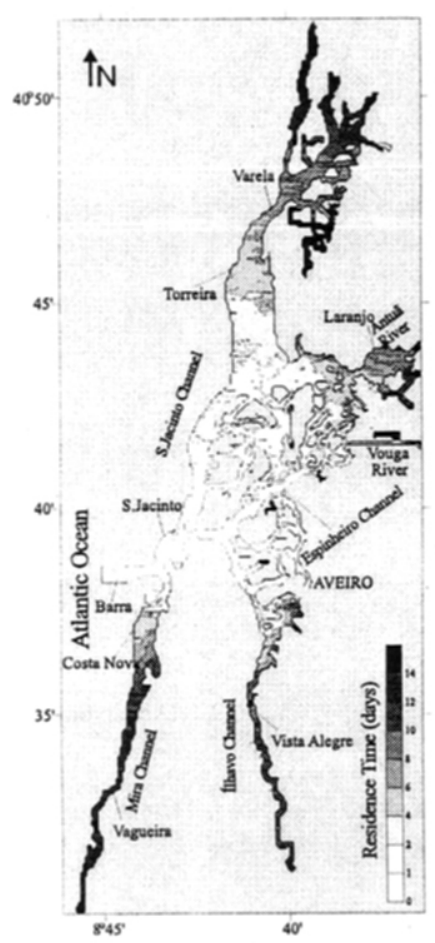

Fig. 6. Contuurs of tidal-mean residence time (days) for passive particles released in each grid cell.

gardless of the phase of the tide at the time of release, although there are some regions where particles did not escape when released into the flood and a few places where none escape regardless of when they were released (e.g. the far end of Mira channel and the dendritic channels close to Varela in the northern area of the lagoon). Residence times less than 2 days are almost entirely confined to the central area of the lagoon (beginning of Mira and Ilhavo channels and almost half of S.Jacinto and Espinheiro channels). The lowest values are found close to the mouth of Ria de Aveiro, where the tidal currents are very strong. Therefore, the water in these areas is rapidly renewed. The residence time pattern determined for Mira and Ilhavo channel is similar, with the values strongly increasing after the first kilometers towards the end of the channels, where they are higher than 14 days. The gradient observed for S.Jacinto channel is much more smoothed, with intermediate values of the order of 1 week found in a large zone close to Torreira. The pattern found for the residence time in Ria de Aveiro clearly reveals the influence of the tidal currents.

The lagoon can be divided in 3 different areas according to the determined residence time pattern. One where the values are lower than 2 days, which is close to the lagoon mouth, and where the influence of the ocean water is very strong and therefore where the water renewal is very good. A second one where the residence tıme is between 2 days and 1 week, which is very small in Ilhavo and Mira channels and that can be identified as a brackish water area. And a third one characterized by residence times higher than 1 week, which is found at the far end of the northern channels and in the last half of Mira and Ilhavo channels, and where the water renewal is small. The residence time pattern found in this study is consistent with the salinity field pattern determined by Dias et al. (1999) for Ria de Aveiro in a summer season, with low river flow into the lagoon. In a system where transport is dominated by longitudinal advection, like in Ria de Aveiro, the salinity is a good tracer for the water renewal during periods of stable conditions. Therefore, these results can be considered representative of the lagoon behaviour in situations of low river flow, which occur during several months in Ria de Aveiro.

\section{Conclusions}

The presented particle tracking model has proved to be a powerful tool for studying dispersion phenomena in Ria de Aveiro. It allows the estimation of the residence time, which can be used to analyze water quality at a low computational cost.

It was concluded that due to the advection mechanism in a time scale higher than 2 tidal cycles almost there isn't mixture between the particles used to characterize each one of the main channels. Therefore, each one of these channels can be considered as an independent estuary with its own characteristics. Considering shorter time scales it is possible to conclude that there is mixture between the particles coming from contiguous channels. Therefore, eventual localized pollution episodes can also have a negative impact in the adjacent channels.

Mira and Ilhavo channels have low residence times only at their first kilometers, being characterized by values higher than 1 week in areas relatively close to the lagoon mouth and by values higher than 2 weeks at the upper reaches of the channels. The residence time in S.Jacinto and Espinheiro channels has a completely different pattern with low values found in most of then areas. Values of about one week are found only above the latitude of $40^{\circ} 45^{\prime}$ and at Laranjo bay, while values higher than 2 weeks are found only at the far end of S.Jacinto channel. Therefore it is possible to conclude that the renew of water in the central area of the lagoon is very good as a reflex of the strong marine influence, while the water renewal is very small at the upper reaches of the channels.

Acknowledgements. The authors thank JNICT/CNRS and PRAXIS XXI, through project EICOS, for financial support granted to this work.

\section{References}

Brooks, D. A., Baca, M. W. and Lo, Y.-T., Tidal circulation and residence time in a macrotidal estuary: Cobscook Bay, Maine Estuarine, Coastal and Shelf Science, 49, 647-665, 1999 
Cheng, R. T., Casulli, V. and Gartner, J. W., Tidal, residual, intertidal mudflat (TRIM) model and its applications to San Francisco Bay, California. Estuarine, Coastal and Shelf Science, 36, 235-280, 1993.

Dias, J. M., Granja, A. P., Lopes, J. F., Dekeyser, I., Circulação Resudual Provocada pela Acção do Vento na Ria de Aveiro, Proceedings do $1^{\circ}$ Simpósio de Meteorologia e Geofísica da APMG $/ 2^{\circ}$ Encontro Luso-Espanhol de Meteorologia, 171$176,1998$.

Dias, J. M., Lopes, J. F., Dekeyser, I., Ilydrological characterization of Ria de Aveiro, Portugal, in early summer. Oceanologica Acta, 22, 473-485, 1999.

Dias, J. M., Lopes, J. F., Dekeyser, I., Tidal propagation in Ria de Aveiro lagoon, Portugal. Physics and Chemistry of the Earth, Part B: Hydrology, Oceans and Atmosphere, 25, 4, 369-374, 2000.

Dronkers, J. J., Tidal computations in rivers and coastal waters. Journal of Hydraulics Division, ASCE, 95, No. HY1, 44-77, 1969.

Falconer, R. A. and Owens, P. H., Numerical modeling of suspended sediment fluxes in estuarine waters. Estuarine, Coastal and Shelf Scuence, 31, 745-762, 1990.

Gomez-Gesteira, M., Montero, P., Prego, R., Taboada, J. J., Leitão, P., Ruiz-Villarreal, M.,Neves, R. and Perez-Villar, V., A two-dimensional particle tracking model for pollution dispersion in A Coruña and Vigo Rias (NW Spain). Oceanologica Acta, 22, 2, 167-177, 1999.
Hofmann, E. E., Hedström, K. S., Moisan, J. R., Haidvogel, D. B. and Mackas, D. L., Use of Simulated drifter tracks to investigate general transport patterns and residence times in the coastal transition zone. Journal of Geophysical Research, 96, C8, 15041-15052, 1991.

Joseph, B. and Swathi, P. S., Lagrangian particle transport in the Indian Ocean: a model study. Journal of Geophysical Research, 104, C3, 5211-5224, 1999.

Leendertse, J. J. and Gritton, E. C., A Water-Quality Simulation Model for Well-Mrxed Estuaries and Coustal Seas, Vol.II. Computational Procedures, The Rand Corporation, New York, 1971.

Marinone, S. G., Effect of the topographic stress on the tideand wind-induced residual currents in the Gulf of California. Journal of Geophysical Research, 103, C9, 18437-18446, 1998.

Moreira, M. H., Queiroga, H., Machado, M. M., Cunha, M. R., Environmental gradients in a southern estuarine system: Ria de Aveiro, Portugal, Implication for soft bottom macrofauna colonization, Neth. J. Aquat. Ecol., 27 (2-4), 465-482, 1993.

Vicente, C. M., Caracterizaçāo hidráulica e aluvionar da Ria de Aveiro, Utilização de modelos hidráulicos no estudo de problemas da Ria, in: Jornadas da Ria de Averro, $I I I$, Edição da Câmara Municipal de Aveiro, Aveiro, Portugal, 41-58, 1985.

Zimmerman, J. T. F., The tidal whirpool: a review of horizontal dispersion by tidal and residual currents, Netherlands Journal of Sea Research, 20, 133-154, 1986. 